\title{
Asegurar la permanencia estudiantil en la Universidad de Costa Rica durante la crisis sanitaria. Reflexiones desde la Vicerrectoría de Vida Estudiantil
}

Ensure student permanence at the University of Costa Rica during the sanitary crisis. Reflection from the Vice Rector Student Office

\section{Volumen 21, Número 3 \\ Setiembre - Diciembre}

pp. $1-20$

Carlos Sandoval García Jose Rivera-Monge

Tatiana Carmona Rizo

Magaly Jiménez Fernández

María Isabel Sánchez Jiménez

Sedali Solís Agüero

Lupita Abarca Espeleta

María del Carmen Rojas Alfaro

Anabelle León Chinchilla

\section{Citar este documento según modelo APA}

Sandoval García, Carlos., Rivera-Monge, Jose., Carmona Rizo, Tatiana., Jiménez Fernández, Magaly., Sánchez Jiménez, María Isabel., Solís Agüero, Sedali., Abarca Espeleta, Lupita., Rojas Alfaro, María del Carmen., León Chinchilla Anabelle. (2021). Asegurar la permanencia estudiantil en la Universidad de Costa Rica durante la crisis sanitaria. Reflexiones desde la Vicerrectoría de Vida Estudiantil. Revista Actualidades Investigativas en Educación, 21(3), 1-20. Doi. 10.15517/aie.v21i3.48165 


\title{
Asegurar la permanencia estudiantil en la Universidad de Costa Rica durante la crisis sanitaria. Reflexiones desde la Vicerrectoría de Vida Estudiantil
}

Ensure student permanence at the University of Costa Rica during the sanitary crisis.
Reflection from the Vice Rector Student Office

\author{
Carlos Sandoval García ${ }^{1}$ \\ Jose Rivera-Monge \\ Tatiana Carmona Rizo \\ Magaly Jiménez Fernández \\ María Isabel Sánchez Jiménez \\ Sedali Solís Agüero \\ Lupita Abarca Espeleta \\ María del Carmen Rojas Alfaro \\ Anabelle León Chinchilla
}

Resumen: La crisis sanitaria producida por el virus SARS-CoV-2 es uno de los retos más complejos de la humanidad en el último siglo. Las universidades públicas enfrentan enormes tareas para asegurar el ingreso, la permanencia, la gestión del plan de estudios y la graduación de la comunidad estudiantil. Este artículo se propone sistematizar y reflexionar sobre las acciones realizadas desde la Vicerrectoría de Vida Estudiantil de la Universidad de Costa Rica, desde mayo hasta diciembre de 2020, como parte de una gestión colegiada en que la coordinación institucional es un elemento vital. Para ello, se analizaron las principales acciones realizadas en un contexto en que las demandas eran inéditas y los tiempos de respuesta, escasos. Se sistematizan algunos de los principales resultados, retos y perspectivas, que podrian ser de retomados en futuros estudios en que se comparen experiencias de otras instituciones de educación superior.

Palabras clave: universidades, covid-19, condiciones de admisión, rendimiento escolar, planes de estudios, beca de estudios, graduación.

\begin{abstract}
The sanitarian crisis produced by the SARS-CoV-2 is one of the most complex challenges faced by humanity in the last century. Public universities encounter enormous tasks for securing admission, retention, curriculum management and graduation of the student community. This article seeks to systematize and reflect on the initiatives undertook by the Vice Rector' Student Office at the University of Costa Rica from May to December 2020 as part of a team work in which institutional coordination was essential. For that purpose, main actions undertaken are analyzed in a context in which demands were unprecedented and time to respond was scarce. In the conclusions, the main outcomes are summarized, which might be considered in further research comparing experiences of other higher education institutions.
\end{abstract}

Keywords: universities, covid-19, admission requirements, academic achievement, curriculum, educational grants, graduates.

\footnotetext{
${ }^{1}$ La información de las personas autoras aparece al final del artículo.

Dirección electrónica de contacto: CARLOS.SANDOVAL@ucr.ac.cr
}

Artículo recibido: 09 de junio, 2021

Enviado a corrección: 24 de junio, 2021

Aprobado: 23 de agosto, 2021 


\section{Introducción}

Este artículo se propone sistematizar y reflexionar sobre algunas de las acciones realizadas desde la Vicerrectoría de Vida Estudiantil de la Universidad de Costa Rica, de mayo a diciembre del año 2020, en el contexto de la emergencia nacional generada por el virus SARS-CoV-2.

Se describen acciones realizadas en términos de asegurar el acceso, permanencia y graduación de personas estudiantes en un periodo particularmente complejo. Para ello, se hace referencia al trabajo llevado a cabo por la Vicerrectoría y las oficinas que la constituyen: Registro e Información (ORI), Becas y Atención Socioeconómica (OBAS), Bienestar y Salud (OBS), y Orientación (OO), así como el Programa de Liderazgo Universitario con Desarrollo Humano y el Programa Voluntariado. En las conclusiones, se repara sobre retos que será necesario acometer en el futuro cercano.

Las acciones llevadas a cabo resultan del trabajo colectivo del personal de Vida Estudiantil en sus oficinas y sedes, en diálogo con el Directorio, el Consejo Superior Estudiantil y asociaciones estudiantiles -especialmente la de las Sedes Regionales, Residencias Estudiantiles, y la de Estudiantes madres y padres- integrantes de la Federación de Estudiantes, que permitió responder a la mayor cantidad posible de retos que la crisis sanitaria fue planteando.

Este artículo se inscribe en lo que se conoce como sistematización de experiencias, es decir, constituye un esfuerzo por pensar sobre acciones que se llevan a cabo en comunidades, organizaciones o instituciones desde la perspectiva de quienes realizamos las labores cotidianas (Jara, 2018). Lo usual es que quienes se ocupan de diseñar políticas o llevarlas a la práctica no alcanzan a reflexionar sobre su puesta en práctica. Esta es una contribución que procura llenar este vacío.

Las acciones realizadas superan con creces lo mencionado en esta contribución, pues se han seleccionado algunas decisiones o acciones que se pusieron en práctica en el contexto de la crisis sanitaria y que podrían servir para realizar, en el futuro, estudios comparados entre instituciones de educación superior a nivel nacional o internacional.

Este artículo se organiza en cuatro secciones. La primera, "Admisión, permanencia y graduación", se ocupa de las acciones realizadas por la Oficina de Registro. La segunda, "Físicamente lejos, afectivamente cerca", presenta iniciativas promovidas desde las oficinas de Orientación y Bienestar y Salud. La tercera, "Análisis como insumo indispensable de la gestión", da cuenta del trabajo de la Unidad de Estudio y Asesoría. La cuarta, "El reto de 
fortalecer el sistema de becas", ofrece un balance de la labor de la Oficina de Becas y Atención Socioeconómica.

\section{Admisión, permanencia y graduación}

El proceso de admisión 2020-2021, que se realiza en conjunto con la Universidad Nacional, fue uno de los principales retos de este periodo. Una primera decisión fue ampliar la fecha de inscripción, originalmente habilitada hasta el 20 de marzo 2020, la cual se extendió hasta el 24 de abril. También se trasladó la fecha de aplicación de la Prueba de Habilidades Cuantitativas $(\mathrm{PHC})$, requisito para algunas de las carreras de las áreas de Ciencias Básicas, Ciencias Sociales, Ingenierías y Salud, y de la Prueba de Aptitud Académica (PAA), a noviembre y diciembre, respectivamente. En la Tabla 1 se presentan las tendencias de personas inscritas en el PAA durante los últimos años. Comparado con el año 2019-2020, la inscripción para el periodo 2020-2021 muestra un ligero descenso al que habría que darle seguimiento en los años por venir.

Estos cambios, a su vez, modificaron las fechas previstas para comunicar los resultados de la PAA, el concurso a recinto y carrera, la solicitud de beca socioeconómica y el inicio del año lectivo 2021, el día 5 de abril. El esfuerzo de organización del Calendario Estudiantil Universitario fue, sin duda, también un reto principal, pues implicaba coordinar procesos y fechas en un periodo más corto. Por primera vez, el calendario, tradicionalmente publicado para personal de la institución de manera impresa, está dispuesto en línea, lo cual es más accesible también para las personas estudiantes ${ }^{2}$.

\section{Tabla 1}

Personas estudiantes inscritas en la PAA 2013-2021

\begin{tabular}{|l|c|c|c|c|c|}
\hline \multirow{2}{*}{ PROCESO } & \multicolumn{5}{|c|}{ CANTIDAD DE ESTUDIANTES } \\
& Inscritos PAA. & Realizaron P.A.A. & Elegibles & Concursantes & Admitidos \\
\hline $2013-2014$ & 40.919 & 34.573 & 20.805 & 11.910 & 8.384 \\
\hline $2014-2015$ & 42.682 & 36.296 & 21.770 & 12.433 & 8.343 \\
\hline $2015-2016$ & 45.654 & 38.334 & 23.544 & 13.399 & 8.383 \\
\hline $2016-2017$ & 48.286 & 40.265 & 24.109 & 14.036 & 9.027 \\
\hline $2017-2018$ & 52.328 & 42.082 & 25.757 & 13.283 & 9.127 \\
\hline $2018-2019$ & 50.906 & 41.337 & 25.489 & 14.570 & 9.327 \\
\hline $2019-2020$ & 57.113 & 46.254 & 27.883 & 16.182 & 9.341 \\
\hline $2020-2021$ & 54.420 & 41.600 & $41.262^{3}$ & 23.818 & 9511 \\
\hline
\end{tabular}

Fuente: Oficina de Registro e Información al 8 de junio del 2021.

2 Se puede consultar en esta dirección https://www.vive.ucr.ac.cr/index.php/calendar

3 La diferencia de 338 estudiantes con respecto a los que hicieron el examen se debe a que a 338 no se les calculó nota, por cuanto no tenían reportado el promedio de Educación Diversificada. Por primera vez, en el año 2021 no existe la nota mínima para ser elegible. 
La realización presencial de la PAA y la PHC fue posible gracias a la aprobación del protocolo por parte del Ministerio de Salud, autoridades con las cuales la coordinación fue muy fluida a pesar de las múltiples demandas que les ha generado la crisis sanitaria. Es de subrayar el trabajo del personal de la OBS en la generación del protocolo de bioseguridad, elaborado conjuntamente con personal del Instituto de Investigaciones Psicológicas (IIP) y el Centro Coordinador Institucional de Operaciones (CCIO). Ello incluyó los lineamientos de limpieza, el protocolo de giras de aplicación y la estimación de las cantidades y costos de los materiales y equipos requeridos.

Hacia finales del año 2020, se gestionó la actualización del domicilio de las personas inscritas, una tarea muy importante, pues pudo haber cambiado debido a la crisis sanitaria y de ello dependía la sede en que les correspondía realizar la PAA. También muy relevante fue la verificación de las calificaciones obtenidas en la Educación Diversificada, pues la nota de admisión se conforma de un 50\% de la PAA y un 50\% justamente del promedio de notas de Educación Diversificada. Se debe subrayar y agradecer la coordinación con el Ministerio de Educación Pública (MEP), que se tradujo en la firma del Convenio de cooperación entre el Ministerio de Educación Pública y las instituciones de educación superior estatal, el cual establece los términos del traslado de las calificaciones obtenidas en secundaria a las universidades públicas.

La preparación de la PAA estuvo acompañada de la Feria Vocacional que, por primera vez, se realizó de manera virtual, del 26 al 30 de octubre, mediante el Facebook live @viveucr. La Feria consistió en sesiones informativas sobre las nueve áreas vocacionales de la UCR y sus respectivas carreras: Bellas Artes, Letras y Filosofía, Educación, Ingeniería, Ciencias Sociales, Ciencias de la Salud, Ciencias Básicas, Ciencias Económicas, Agropecuarias y Forestales; actividades de orientación vocacional sobre temas de interés para las personas aspirantes como: análisis de intereses y aptitudes, aspectos a considerar para la información de carreras y la construcción de planes de acción para la toma de decisiones, vida universitaria, servicios de la UCR, proceso de admisión, becas entre otros.

De forma complementaria, a partir del 12 de octubre se habilitó el sitio web: https://feriavocacional.ucr.ac.cr/, el cual contiene información y materiales de apoyo sobre la oferta académica de la UCR, planes de estudio, recintos y sedes donde se imparten las carreras, los cortes históricos de ingreso, el perfil de salida profesional, requisitos especiales, entre otros. Además, incluyó productos audiovisuales que contribuyeran a guiar a la persona aspirante en torno a preguntas, tales como: por qué estudiar en la UCR, sistema de becas, 
proceso de admisión y elección de carrera, así como las grabaciones de todas las actividades sincrónicas que se realizaron en la Feria Vocacional Virtual (FVV). La FVV, celebrada del 26 al 30 de octubre 2020, tuvo un alcance de 133020 personas y logró 23527 interacciones, las cuales incluyen "me gusta", comentarios y números de veces en que las publicaciones fueron compartidas. Asimismo, 7236 personas participaron en los Facebook live y hubo 6804 visitas a la página.

Para apoyar la atención de consultas, se desarrolló un ChatBot, un robot preparado a responder algunas de las preguntas frecuentes que suelen formular las personas estudiantes y que permanecerá como un recurso en la Universidad para la atención de consultas, administrado desde la Oficina de Divulgación e Información (ODI).

En términos de la matrícula, resalta el hecho de que el dato de estudiantes matriculados en el segundo ciclo lectivo 2020 constituye la mayor cantidad en un segundo ciclo lectivo en la historia de la Universidad de Costa Rica, tal y como se aprecia en la Figura 1.

Figura 1.

Cantidad de estudiantes matriculados por año y ciclo lectivo por sede y recinto 2017-2020

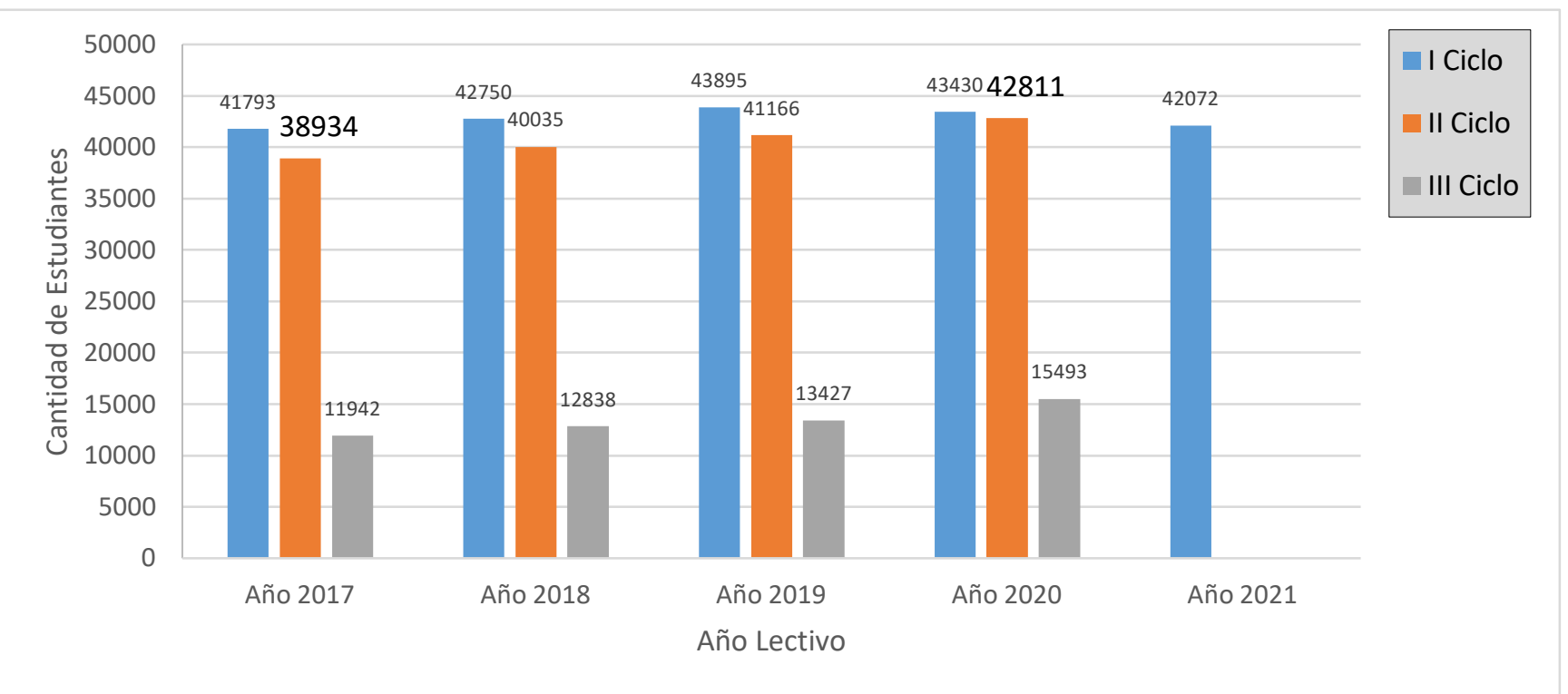

Fuente: Oficina de Registro e Información a diciembre 2020.

En medio de las dificultades de la crisis sanitaria, este dato muestra que la virtualidad tiene potencialidades que, acompañadas de conectividad, en términos de señal como de capacidad de pago, pueden favorecer procesos de acceso, permanencia y graduación. 
Durante el año 2020 se entregaron 5484 títulos, que representa un $7 \%$ menos que los entregados en el año 2019, una diferencia sobre la cual se requiere mayor análisis. En el mes de abril de 2020, se utilizaron los servicios de Correos de Costa Rica vía convenio con la Sección de Correos de la UCR. Para los periodos de graduación correspondientes a los meses de junio, agosto, octubre y diciembre de 2020 , se recibió la colaboración de la Sección de Transportes y de Correos de la UCR, a través de la flotilla de transportes de la Universidad, para la entrega de los diplomas.

\section{Físicamente lejos, afectivamente cerca}

En este periodo, la Oficina de Orientación facilitó atención individual especializada a la población estudiantil, en las áreas de Orientación, Psicología y Trabajo Social, a un total de 2235 estudiantes en 3917 sesiones desarrolladas. Esta atención individual se abocó a dar respuestas a necesidades de tipo personal, académico, vocacional - ocupacional, socioeconómico y de accesibilidad de la población estudiantil universitaria.

También se trabajó en una estrategia de bienvenida al II ciclo, 2020 con estudiantes de nuevo ingreso (carné C0). Alrededor de 5564 estudiantes de todas las Sedes y Recintos de la Universidad recibieron por correo electrónico el video y la presentación de las actividades.

El personal de la Oficina de Orientación desarrolló actividades grupales y envío de material de apoyo académico a la población estudiantil. Estas actividades pusieron especial énfasis en temas vinculados con los cambios generados por el desarrollo de los cursos de manera virtual. Hacia finales de 2020, un total de 61832 personas accedieron a los materiales.

Para el II ciclo, se diseñó una estrategia de acompañamiento virtual dirigida a la población estudiantil en general, denominada "Orientación hasta tu casa", en la cual semanalmente se abordaron diferentes temáticas que respondieron a las necesidades detectadas y manifestadas por las personas estudiantes.

Dicha estrategia incluyó actividades sincrónicas y asincrónicas, las cuales brindaron recursos informativos y herramientas para el trabajo a nivel personal, académico y vocacionalocupacional ante la continuación de un II ciclo lectivo virtual. Estos recursos se compartieron por medio de transmisiones sincrónicas, difusión de videos y audios, envío de material multimedial y un repositorio digital de materiales. Entre mayo y diciembre de 2020, con las actividades asincrónicas, 190175 personas fueron alcanzadas mediante el Facebook de la Oficina de Orientación y se registraron 924 visualizaciones en el canal de YouTube. 
Las actividades tuvieron un alcance general de 27035 personas, de las cuales 1080 asistieron de manera sincrónica, y 25955 accedieron reproduciendo el material que, posterior a la transmisión de video, se compartió en las redes sociales y en la página web de la Oficina de Orientación.

Por su parte, a partir del 19 de marzo del 2020, el área de psicología de la OBS realizó una transición completa de consulta presencial al servicio de teleconsulta psicológica mediante la plataforma de videoconferencias Zoom, con lo cual se logró mantener la oferta de atención psicológica a las personas usuarias regulares del servicio. De enero a diciembre del 2020, se realizaron 2147 consultas individuales. Estas atenciones han incluido a más de 250 personas tanto de la población estudiantil como personas funcionarias.

El servicio de teleconsulta se vio apoyado por el Proyecto Mishka, que en bribri significa "vamos" (Jara y García, 2013), una iniciativa colectiva de profesionales en salud mental de la Universidad de Costa Rica que, de forma voluntaria, acompañaron emocionalmente, mediante una intervención en crisis, a personas de la comunidad universitaria que se vieron afectadas psicológicamente por la pandemia COVID-19.

El proyecto Mishka nació el 27 de abril 2020, ante una imperiosa necesidad de ofrecer un espacio de acompañamiento y escucha ante el malestar emocional que se estaba agravando como consecuencia de la pandemia. El propósito fue fomentar la estabilidad emocional, procurar el afrontamiento propositivo y motivar la búsqueda de apoyo social. Los pilares de intervención son la escucha activa, la expresión de emociones y sentimientos, la regulación cognitiva, las modificaciones conductuales y el reforzamiento de redes de apoyo. La estrategia de intervención se configuró dentro de la estructura de una intervención en crisis que se basa en un asesoramiento comprensivo de los factores de riesgo que puedan conllevar a problemas psicológicos, tales como: malestar emocional, duelo, autolesiones o posible daño a terceros, ansiedad, depresión, crisis de pánico, separación familiar, entre otros.

Hasta noviembre de 2020 más de 140 personas se vieron beneficiadas con el proyecto. En promedio, cada persona recibió cuatro sesiones, lo que representa 560 consultas. Los procesos de atención psicológica grupal fueron una segunda forma de intervención, los cuales se fundamentaron en los principios de la terapia grupal tanto a nivel psicoterapéutico como de grupos de apoyo, que se configuran como un espacio para compartir y construir herramientas que posibiliten a las personas participantes el intercambio de experiencias. Los grupos de apoyo constituyeron una herramienta esencial para el fortalecimiento de los vínculos y la revitalización del lazo social en un contexto de crisis. Estos espacios grupales se convirtieron, ya de por sí, en 
una alternativa eficiente en la construcción de herramientas y en el fortalecimiento de la capacidad de generar vínculos de las personas integrantes. En la estrategia grupal se han dedicado 902 horas consulta. Por lo que a nivel general (individual y grupal) se han brindado 2601 horas de consulta.

Este periodo tan complejo estuvo también caracterizado por un esfuerzo por mejorar las herramientas de comunicación digital de la Vicerrectoría de Vida Estudiantil, cuyo perfil de Facebook pasó de 12702 en mayo a 21072 personas seguidoras en octubre 2020, lo cual representa un crecimiento importante. Este aumento se vio complementado con el alcance, pues, en mayo, las publicaciones solían tener, en promedio, un alcance de 1100 personas y, para el 12 de junio, por ejemplo, se logró un alcance de 79000 personas, una de las cifras más altas. En octubre, la página tuvo un alcance global de 472778 personas, lo cual refleja un incremento del $512 \%$ con respecto al mes de mayo. Estos incrementos se acompañaron también de la apertura de una cuenta en Instagram, particularmente relevante para interactuar con las nuevas generaciones.

De igual relevancia fue la apertura de perfiles de Facebook e Instagram de la Oficina de Registro e Información, en ocasión de su 65 aniversario. En mayo de 2021, el perfil de Facebook de la ORI reporta 12000 personas vinculadas, lo cual sugiere una recepción positiva por parte de la comunidad estudiantil. Los perfiles de la ORI en Facebook e Instagram se complementan con los disponibles desde la Oficina de Orientación, que cuenta con 50000 personas asociadas.

No se podría dejar de mencionar que durante el segundo ciclo 2020 , la ORI y la OBAS pusieron a disposición sus datos en la página de Transparencia de la UCR, con lo cual se cuenta con indicadores sociodemográficos anonimizados de las personas que cursan estudios en la Universidad de Costa Rica y su respectiva beca, ello permite identificar perfiles por carrera, sede de estudios, género, entre otras variables. Esta decisión contribuye por una parte, a contar con perfiles de la población estudiantil a partir de datos empíricos y, por la otra, da cuenta de cómo se invierten los recursos que aporta la sociedad costarricense.

\section{Análisis como insumo indispensable de la gestión}

La Unidad de Estudio y Asesoría (UEA) de la Vicerrectoría constituye una instancia de asesoría y gestión. Se trata de combinar las acciones de gestión con un esfuerzo reflexivo y analítico, una combinación compleja especialmente en el contexto de la crisis sanitaria, pues 
las demandas aumentan y los tiempos de respuesta se acortan. En este marco, vse realizaron varios estudios en el periodo considerado en este artículo.

En el marco del fortalecimiento de la permanencia de la población estudiantil en las actividades académicas, la UEA desarrolló tres estudios sustantivos con temáticas vinculadas al proceso de permanencia, cuyas conclusiones principales se sintetizan enseguida.

Uno de ellos se tituló Análisis de los datos de matrícula consolidada de la población estudiantil de pregrado y grado en el l ciclo lectivo 2020, por rendimiento académico obtenido y carga académica, según el cumplimiento parcial de los parámetros para el concurso de traslado e ingreso a carrera por las modalidades de rendimiento académico y excelencia académica. Algunos de los hallazgos y conclusiones principales sobre matrícula y rendimiento académico de las personas estudiantes fueron los siguientes.

En el I ciclo lectivo del 2020, un 52,90\% (22.191) de personas estudiantes matriculó una carga académica (CA) de 12 créditos o más. De este grupo, un 33,83\% (14.188) obtuvo un rendimiento académico (RA) de 8.5 o más, mientras que en el caso del I ciclo lectivo 2019, este comportamiento es del 24,71\% (10.441 personas estudiantes).

En este contexto, del I ciclo lectivo 2020, un 21,37\% (8.964) se estima como potenciales participantes en el concurso de traslado e ingreso a carrera por la modalidad de RA del año 2021, con CA de 12-14 créditos matriculados y RA de 8.5 o más, o CA de 15 créditos o más, y RA entre 8.50 y 8.99 , y en el I ciclo lectivo 2019 , el $16,69 \%$ (7.053 personas estudiantes) contó con estas condiciones.

Asimismo, un 12,45\% (5.224) se estima como potenciales participantes en el concurso de traslado e ingreso a carrera por la modalidad de Excelencia Académica (EA) del año 2021, con una CA de 15 créditos o más matriculados y R.A. de 9,00 o más, y en el I ciclo lectivo 2019 un 8,02\% (3.388 personas estudiantes) contó con estas condiciones.

En el I ciclo lectivo 2020, el porcentaje de personas estudiantes con promedio ponderado, igual o mayor que 8,50 con 12 o créditos o más, es mayor en 9 puntos porcentuales que en el año 2019, lo que pone de manifiesto que el proceso de permanencia en el contexto de la pandemia por el virus SARS-CoV-2 contuvo y fortaleció las condiciones para aplicar los parámetros académicos definidos para el concurso de traslado e ingreso a carrera del año 2021.

El segundo estudio se tituló Comportamiento de la Demanda Insatisfecha, en pregrado y grado, 2018-2020 (ViVE-1666-2020). Para el I ciclo lectivo de los tres años estudiados, el porcentaje promedio de cupos asignados, con respecto a los cupos ofrecidos, es del $80 \%$ 
para matrícula ordinaria, 23\% para inclusión y 10\% para aprovechamiento de cupos. Los datos correspondientes al II ciclo lectivo son de $78 \%, 22 \%$ y $7 \%$, respectivamente.

En el caso del I ciclo lectivo de los tres años estudiados, el porcentaje promedio de cupos de demanda insatisfecha por falta de cupo es cercano al 13\% para matrícula ordinaria, 18\% para inclusión y 5\% para aprovechamiento de cupos. Para el II ciclo lectivo del período examinado, la demanda insatisfecha por falta de cupo es cercana, en promedio, al 10\% para matrícula ordinaria, $11 \%$ para inclusión y $3 \%$ para aprovechamiento de cupos.

Cuando se desagrega por tipo de curso se observa que la mayor parte de los cursos con alta cantidad de cupos de demanda insatisfecha son cursos complementarios, de servicio o mixtos, lo cual permite comprender dónde radican los retos principales. Se podría sugerir la posibilidad de ofrecer cursos de alta demanda, usualmente de servicio, de manera virtual, con la producción de materiales de apoyo, los cuales estarían disponibles previo al inicio de las lecciones y serían de acceso público.

El tercer estudio se titula Estudio del comportamiento de Retiro de Matrícula (RM), I ciclo 2014 -2020 y II ciclo 2014 - 2019. Este estudio está precedido por un cambio en el artículo 27 del Reglamento Régimen Académico Estudiantil, el cual consistió en disminuir el periodo dispuesto para el retiro de matrícula: de las primeras 4 semanas de clases para los I y II ciclos lectivos y la primera semana de clases en el III ciclo lectivo, a los primeros 6 días de la primera semana de clases en los I y II ciclos lectivos y los primeros 2 días de clases en el III ciclo lectivo, respectivamente.

Al respecto, para el I ciclo lectivo del año 2018, de los de 9139 cupos disponibles por RM se ofertaron un aproximado de $4133(45,2 \%)$ en matrícula de aprovechamiento, mientras que para el I ciclo 2019, se lograron ofertar los 4426 (el 100\%). En cuanto al II ciclo lectivo 2017, de 8105 cupos liberado por RM se ofertaron un aproximado de 3171 (un 39,1\%) cupos de RM en matrícula de aprovechamiento, y en el II ciclo 2018, los 3681 (100\%) de los cupos liberados por RM. Una conclusión particularmente relevante es que se observa un mayor aprovechamiento de cupos en la Matrícula Ordinaria, al revertirse la tendencia de "matricular y luego retirar", y su incidencia en las posibilidades de avance en el plan de estudios de la población estudiantil.

Un cuarto estudio del que la UEA se ocupó fueron las Acciones relacionadas con los períodos de cancelación de los aranceles de matrícula, y supresión de los recargos del 10\% y $20 \%$, en el contexto de la pandemia por SARS-CoV-2, como parte de una labor común entre las oficinas de Becas y Atención Socioeconómica, Registro e Información, Administración 
Financiera y el Sistema de Estudios de Posgrado, tendiente a introducir ajustes y adecuaciones de distintas actividades universitarias para asegurar la articulación de los procesos favorecedores de la permanencia de la población estudiantil. Ello implicó la modificación de los plazos para la cancelación de los aranceles del I ciclo lectivo 2020, con el propósito que las personas estudiantes pudieran realizar la matrícula del II ciclo lectivo 2020 dentro del plazo para esta cancelación (ViVE, 2020a).

También se introdujo una modificación del plazo de las fechas máximas de cancelación de los aranceles de matrícula correspondientes al I, II y III ciclos lectivos 2020, para que las personas estudiantes de Grado y Posgrados Regulares, Posgrados con Financiamiento Complementario (modalidad semestral) y Posgrados con Financiamiento Complementario (modalidad no semestral) dispusieran de un plazo mayor para la cancelación (ViVE, 2020b; ViVE, 2020c).

De manera armónica, el plazo de recepción de solicitudes del beneficio de pago como nacional se amplió en un mes calendario para el I ciclo lectivo 2020. Este beneficio permite a las personas estudiantes de nacionalidad extranjera realizar el pago de matrícula de acuerdo con los aranceles de cobro que aplican para las personas estudiantes costarricenses.

Este cuarto estudio facilitó, también, la eliminación de los recargos del $10 \%$ y $20 \%$ sobre los aranceles de matrícula del I, II y III ciclos lectivos 2020, gestionada ante el Consejo Universitario como una facilidad para la población estudiantil en las circunstancias de la crisis sanitaria (Consejo Universitario, 2020a, Consejo Universitario, 2020b).

Por último, una quinta iniciativa a cargo de la UEA, que relaciona las gestiones de distintas unidades académicas ante la Rectoría y las vicerrectorías de Docencia y de Vida Estudiantil, se dirigió a identificar, de manera conjunta con las oficinas de Becas y Atención Socioeconómica y de Registro e Información, el mecanismo para que las personas estudiantes con matrícula consolidada en cursos-grupos del I ciclo lectivo 2020, que las unidades académicas no podían finalizar por sus componentes prácticos, pudieran completarlos en un momento diferido. Esta ruta de análisis implicó determinar alternativas que combinaran la prevención de afectaciones negativas en el rendimiento académico y posibilitara el otorgamiento de los beneficios del Sistema de Becas en el período en que estos se completaran, según se plasma en la Resolución ViVE-12-2020 del 02 de julio de 2020 (ViVE, 2020d).

Esta decisión es aún más apremiante en las carreras del Área de la Salud, lo cual implica un monitoreo constante para las previsiones que se requieren implementar en la ORI y en 
OBAS tanto para la activación de los cursos-grupos (se ha denominado "Procedimiento retorno COVID") como para el otorgamiento de los beneficios del Sistema de Becas. Sin embargo, más allá, amerita de un esfuerzo de la Universidad y de las unidades académicas para acompañar a las personas estudiantes ante una inevitable postergación en el avance de su plan de estudios.

\section{El reto de fortalecer el sistema de becas}

La Universidad de Costa Rica destina un $11 \%$ de su presupuesto al sistema de becas. ${ }^{4}$ En el I ciclo lectivo 2020, 23445 estudiantes (54,7\%) contaron con alguna categoría de beca socioeconómica. En las Sedes Regionales esta cobertura alcanza un 78,0\% (en la Sede Sur un $94,8 \%$ ) y en la Sede Rodrigo Facio representa un 46\%. Del total de la población estudiantil con beca socioeconómica vigente en el primer ciclo lectivo del año 2020, el 17\% recibió beca 1, 2 o 3 (3 959 estudiantes) y el 83\% tuvo la categoría de beca 4 o 5 (19 486 estudiantes). Es importante anotar que las categorías de becas 4 y 5 otorgan beneficios socioeconómicos, entre los que se incluyen: alimentación, transporte y alojamiento (reubicación geográfica 0 residencias estudiantiles). En el II ciclo lectivo 2020 (hasta octubre), se asignó la beca por actividades universitarias a un total de 6300 (6587 en el primer ciclo 2020) estudiantes. Se cuenta también con un número de acuerdos con instituciones o personas donantes que permiten ofrecer las becas: Clyde J. Surgi, Crowley, Victoria Salas D., Golcher Barguil, Guido Sibaja, Construyendo Oportunidades: Colegio Federado de Ingenieros y Arquitectos, Elsa Carranza Zamora, y Yolanda Avilés Rayo.

Durante el II ciclo lectivo 2020, la OBAS fortaleció el monto asignado por concepto de almuerzo y de otros tiempos de alimentación en un 30\%, lo cual incluye a las personas becarias 3 (reciben un $70 \%$ del beneficio de almuerzo), 4 y 5, que constituye el $90 \%$ del estudiantado con beca socioeconómica. También se aumentó un 15\% el beneficio del monto económico de gastos de carrera, lo cual incluye a las personas becarias 5 .

En el contexto de la pandemia, en lo que respecta a la reubicación geográfica (RG), en cuanto al lugar del alquiler por parte de la persona estudiante, para el II y III ciclo lectivo 2020, si la persona estudiante cumplía con las condiciones de categoría de beca, y geográficas para el disfrute de este beneficio, podía elegir el lugar para el alquiler de manera independiente al

\footnotetext{
${ }^{4}$ No se cuenta con datos sobre inversión en becas en otras instituciones de educación superior, que permita poner en perspectiva comparada el presupuesto en becas de la Universidad de Costa Rica. En el futuro sería del tipo de investigaciones por llevarse a cabo, ojalá con una perspectiva regional en América Central o América Latina.
} 
campus universitario donde estudiara. Bastaba que el requerimiento de alquiler se constituyera en elemento esencial para poder continuar estudiando bajo la modalidad virtual (OBAS, 2020b).

Aunque no pertenece al sistema de becas, en el contexto de la crisis sanitaria, se entregó un apoyo de conectividad de 15.000 colones a las personas becarias 4 y 5 , alrededor de 19 000 personas, durante el segundo ciclo 2020, a fin de cubrir los costos de internet en un contexto de virtualidad (Rectoría, 2020a). Estos apoyos se mantuvieron para el III ciclo 2020 (R-12-2020b).

El antecedente fue la entrega de tabletas que inició la administración del anterior rector, el Dr. Henning Jensen Pennington, la cual se continuó durante el segundo semestre 2020, en total se entregaron 3000 tabletas adicionales a lo largo del año. Además, se introdujo un sistema de solicitud en línea, disponible en el Portal UCR, lo cual permite la trazabilidad.

Tanto la directriz sobre Reubicación geográfica como la de apoyo de conectividad procuraron responder a lo manifestado por estudiantes y docentes de sedes regionales durante las reuniones de trabajo llevadas a cabo durante junio de 2020. Las visitas de trabajo a las sedes regionales (excepto la de Guanacaste debido al cierre sanitario) fueron particularmente importantes para documentar las consecuencias de la crisis sanitaria para las personas estudiantes. Luego de las visitas se determinó consolidar un espacio mensual de reunión con las personas coordinadoras de Vida Estudiantil de las sedes regionales, que permitió recoger recomendaciones y mejorar los niveles de coordinación y la capacidad de respuesta.

Para el año 2021, el presupuesto de becas mantuvo el mismo monto asignado en el año 2019 y fue una de las pocas partidas del presupuesto universitario que no disminuyó. Este escenario financiero debe tomar en cuenta varios factores. Uno es que el superávit de este año se estima en 800 millones de colones, cerca del 3\% del total del presupuesto de becas. Un decreto por publicarse por parte del gobierno de la República permitirá re-presupuestar superávits en becas, con lo cual este monto se podrá emplear en el año 2021.

Si la virtualidad se extendiera para todo el I ciclo 2021 y, más aún, para el segundo II ciclo 2021, se requerirían montos estimados en el oficio OBAS-1067-2020 (OBAS, 2020a). Es decir, los requerimientos financieros variarán según se modifique o no la virtualidad, un proceso al que habría que darle seguimiento muy cuidadoso.

EI acuerdo FEES incluye que el gobierno de la república reconocería un $5 \%$ aún no presupuestado. Si esto se pudiera concretar constituiría un ingreso adicional para la 
Universidad, que podría emplearse para financiar conectividad. Sin embargo, mucho dependerá de la situación macroeconómica del país durante el año 2021.

Para el segundo ciclo lectivo del año 2020, la OBAS asumió el reto de virtualizar el 100\% del proceso de recepción de solicitudes de beca socioeconómica y, desde el mes de junio, en conjunto con el Centro de Informática, se trabajó en la virtualización de los procesos de solicitud y revisión de formularios de beca socioeconómica, lo cual permitió la recepción del $100 \%$ de la documentación de respaldo de la condición socioeconómica.

Junto con el trabajo que realizan las oficinas, la Vicerrectoría impulsa los programas de Liderazgo y Voluntariado, que se enmarcan como oportunidades de crecimiento para el estudiantado. Comparten la importancia de impulsar iniciativas ciudadanas de parte de la comunidad estudiantil que promuevan actitudes propositivas ante los grandes retos que vivimos.

Ambos programas comparten también la importancia del trabajo cooperativo entre quienes estudian en diferentes disciplinas, pero que comparten interrogantes comunes frente a desafíos comunes, como puede ser, por ejemplo, la crisis climática. Desde el Programa de Liderazgo, un total de 2434 estudiantes participaron en actividades de capacitación, formación, proyección, sensibilización y promoción. Mientras tanto, se contabilizaron 2307 participaciones estudiantiles en proyectos virtuales gestionados por el Programa de Voluntariado.

El Programa de Liderazgo Universitario con Desarrollo Humano orientó sus esfuerzos en capacitar virtualmente al estudiantado en temas de liderazgo, inteligencia emocional y desarrollo humano. Se procuró facilitar herramientas que permitieran desarrollar auto liderazgo y bienestar, vinculado, al tema de sostenibilidad, desde casa. Con estos grandes objetivos también se ofreció el primer campamento virtual Lidera-T en el tercer ciclo lectivo.

Se realizaron las campañas "Lidera tu bienestar" y "Hogares sostenibles y sostenibilidad" a través de redes sociales para acompañar a los estudiantes y seguirles ofreciendo herramientas de afrontamiento, liderazgo y desarrollo, que les permitieran alcanzar sus objetivos y minimizar el impacto de los cambios de la vida académica, social y familiar. Una tercera campaña estuvo orientada a destacar el liderazgo de la Universidad de Costa Rica a nivel social, en su 80 aniversario, para reforzar la importancia de esta Institución a nivel nacional e internacional, y motivar a los estudiantes ante los cambios acontecidos por la pandemia COVID19.

Se orientaron las acciones con el acompañamiento de los estudiantes facilitadores activos y estudiantes graduados, quienes abocaron sus esfuerzos en pro de contribuir con las 
personas estudiantes a superar las condiciones actuales y a motivarlos para la adaptación de los cambios con flexibilidad y positivismo. Este acompañamiento, desde los pares, a través de videos, espacios de integración y formación fue muy importante y con una respuesta muy positiva de parte de los estudiantes participantes.

Las actividades que realiza el Programa de Voluntariado, históricamente y según se concibe desde su creación, han estado enfocadas en proveer espacios de formación integral y fortalecimiento de habilidades blandas para la vida de las y los estudiantes. Este objetivo es medular en el quehacer del Programa y es por esto que, en este contexto tan incierto y convulso, se crean espacios complementarios a la virtualización académica donde las y los estudiantes se inscribieran de manera voluntaria. Los datos de participación y los comentarios de las y los estudiantes confirman que estos espacios de proyección de la imaginación creativa, aprendizaje voluntario y creación de conocimiento conjunto se tradujeron en bienestar físico y mental.

A pesar de los retos que conlleva la virtualidad como una nueva forma de accionar desde el voluntariado, se llevaron a cabo iniciativas muy valiosas a lo largo del año entre las cuales podemos mencionar Talleres de Primeros Auxilios Psicológicos, apoyo al Programa de Integración de la Persona Adulta y Adulta Mayor (PIAM), Taller ManejándoME y a mis estudiantes en tiempos de COVID-19, el cual tuvo como objetivo trabajar con docentes de primaria y secundaria el manejo adecuado del estrés y de las emociones por medio de una intervención corta de entrenamiento en estrategias de afrontamiento y autorregulación basadas en evidencia. Nueve centros educativos públicos en los cantones de Desamparados y Alajuelita en San José y el cantón de Osa en Puntarenas participaron en esta iniciativa.

Este tiempo invertido también permitió que las personas voluntarias tuvieran un espacio de crecimiento personal, contribuyendo con una formación humanística en un contexto de esparcimiento, de adquisición de nuevas habilidades y de promoción de salud mental.

\section{Retos y perspectivas}

En esta última sección se retoman algunos aprendizajes y retos de alcance institucional que requerirán de la reflexión y de la acción en los años por venir. Un primer aprendizaje es que el trabajo coordinado entre las oficinas, la Unidad de Estudio y Asesoría, y el personal de Vida Estudiantil de las sedes regionales es particularmente importante, más aún, en el contexto de la crisis sanitaria, la cual demanda combinar reflexión y toma de decisiones en periodos cortos. Buena parte de este vínculo se materializa en el Calendario Estudiantil Universitario, el 
cual constituye, por decirlo así, la partitura de muchas actividades de la Institución. En el año 2020, se introdujeron 9 modificaciones. El trabajo de coordinación en torno a estos cambios ha producido un agotamiento del personal de la Vicerrectoría que debe atenderse cuidadosamente durante este año 2021.

La Oficina de Registro e Información cumplió 65 años, como ya se anotó y, sin duda, uno de los retos principales radica en la modernización del Sistema de Aplicaciones Estudiantiles (SAE). EI SAE está construido a partir de un lenguaje llamado power builder, ya muy poco empleado en sistemas de información. El Comité Gerencial de Informática tomó el acuerdo de iniciar los trabajos de un nuevo sistema de información en el año 2019, pero no se puso en práctica. Este un reto cardinal de la ViVE para los próximos 4 años.

La Prueba de Aptitud Académica (PAA) fue, posiblemente, el reto más importante de la Vicerrectoría de Vida Estudiantil a lo largo del segundo ciclo 2020. Gracias al esfuerzo de varias dependencias y del personal pudimos cumplir los objetivos trazados. Para los años por venir se requiere prestar particular atención a las solicitudes de apertura de sedes de aplicación por parte de instituciones de secundaria de zonas rurales, algo a lo que el Instituto de Investigaciones Psicológicas (IIP) debe prestar atención. Además, se requiere que la Comisión de Admisión evalúe la factibilidad y conveniencia de realizar la PAA en forma virtual. Para ello, el trabajo coordinado con el Centro de Informática será de vital importancia.

La reunión mensual con las personas coordinadoras de Vida Estudiantil de las sedes regionales permitió comprender los desafíos que viven las personas estudiantes con menores recursos y oportunidades. De especial relevancia, en el contexto de las sedes, es el hecho de que mientras la población estudiantil de sedes y recintos ha crecido, el personal de Vida Estudiantil se mantiene. Ello ha producido un incremento de la demanda de servicios que requiere ser atendida.

Estas reuniones también permitieron reconocer que el acceso a internet es un problema en diversas regiones del país, lo cual condiciona el empleo de las tabletas y del mismo apoyo para conectividad. Por ello, en coordinación con el Centro de Informática, diseñamos una encuesta mediante la cual las personas estudiantes con dificultades de acceso a internet geolocalizaron su residencia. Esta información le permitiría al Instituto Costarricense de Electricidad determinar las posibilidades de facilitarles señal. El resultado final de esta superposición de mapas - alcance de la red Kolbi y puntos de difícil conectividad- es de vital importancia. 
El encuentro y coordinación de iniciativas entre las oficinas de Orientación y Bienestar y Salud es una tarea que requiere también seguimiento. El reconocimiento de la promoción de la salud y de sus principales determinantes es una perspectiva compartida por ambas oficinas, cuyas tareas se complementan y pueden encontrar sinergias que potencien sus labores.

De igual importancia es el seguimiento a los vínculos entre los procesos de orientación vocacional y el proceso de admisión. Ambos procesos son retadores y complejos tanto para las personas estudiantes como en términos del acompañamiento que pueda ofrecer la Institución. Los cambios tecnológicos en curso y las posibilidades de empleabilidad, entre otros retos, plantean nuevas preguntas a la orientación vocacional, que requieren ser atendidas posiblemente no solo en el último año de educación secundaria, sino desde antes.

La coordinación con las otras universidades públicas, en el marco del Consejo Nacional de Rectores, es también un reto que se requiere profundizar. Las comisiones de trabajo, vigentes ya por años, de pronto se deberían revisar, de modo que se determine si responden a los escenarios actuales. El año 2020 reveló diferentes modalidades de admisión sobre las cuales también debería haber análisis en términos de sistema público de universidades. En una perspectiva más amplia, las universidades públicas tienen el reto de constituirse en un sistema de educación superior que, como tal, podría potenciar su contribución a la sociedad costarricense.

Por último, el curso de la crisis sanitaria confirma la enorme complejidad de estos tiempos, caracterizados por avances y retrocesos en términos de casos, letalidad y mortalidad. Más que una tendencia, si se quiere lineal hacia la llamada "post pandemia", los datos confirman que la Institución y, en general, el país, enfrenta enormes retos en el campo educativo, lo cual incluye garantizar la permanencia y procesos de enseñanza y aprendizaje idóneos en contextos de virtualidad. Incluso, cabría plantearse si la Institución tendrá que incrementar el presupuesto dedicado a becas, actualmente en un 11\%, como una forma de responder a la crisis económica que ha incrementado el desempleo, la pobreza y la desigualdad. Se trata de tiempos muy difíciles frente a los cuales se requieren políticas de gran calado. 


\section{Referencias}

Consejo Universitario. (2020a). Sesión N 6397, artículo 5, del 25 de junio de 2020. Recuperado https://www.cu.ucr.ac.cr/uploads/tx ucruniversitycouncildatabases/minute/2020/6397.pdf

Consejo Universitario. (2020b). Sesión $N^{\circ}$ 6429, artículo 6, del 1 de octubre de 2020). Recuperado https://www.cu.ucr.ac.cr/uploads/tx ucruniversitycouncildatabases/minute/2020/6429.pdf

Jara Holliday, Oscar. (2018). La sistematización de experiencias: práctica y teoría para otros mundos políticos. Bogotá: Centro Internacional de Educación y Desarrollo Humano CINDE. Recuperado de https://cepalforja.org/sistem/bvirtual/?p=1689

Jara Murillo, Carla Victoria. y García, Alí. (2013). Se’ ttö bribrie ie = Hablemos en bribri. San José: Ed. Digital. Recuperado de https://www.lenguabribri.com/se-tt\%C3\%B6-bribri-iehablemos-en-bribri

Oficina de Becas y Atención Socioeconómica (OBAS). (2020a). Oficio 1067. Disponible en la Vicerrectoría de Vida Estudiantil.

Oficina de Becas y Atención Socioeconómica (OBAS). (2020b). Circular OBAS 6. Recuperado de https://becas.ucr.ac.cr/wp-content/uploads/2020/07/Circular-OBAS-6-2020.pdf

Rectoría de la Universidad de Costa Rica. (2020a). Resolución 327. Recuperado de http://www.rectoria.ucr.ac.cr/site/wp-content/uploads/2020/12/resolucion-r-327-2020.pdf

Rectoría de la Universidad de Costa Rica. (2020b). Resolución R-12-2020. Recuperado de http://www.rectoria.ucr.ac.cr/site/wp-content/uploads/2020/12/resolucion-r-327-2020.pdf

Vicerrectoría de Vida Estudiantil (ViVE). (2020a). Modificación V a la Resolución VIVE-1-2020. Recuperado de https://www.vive.ucr.ac.cr/index.php/node/index/394

Vicerrectoría de Vida Estudiantil (ViVE). (2020b). Modificaciones VI a la Resolución VIVE-12020.

Recuperado

de https://www.vive.ucr.ac.cr/uploads/document/410 5f10a98c51d0a.pdf

Vicerrectoría de Vida Estudiantil (ViVE). (2020c). Modificación IX a la Resolución VIVE-1-2020. Recuperado de https://www.vive.ucr.ac.cr/index.php/node/index/455

Vicerrectoría de Vida Estudiantil (ViVE). (2020d). Resolución 12. Recuperado de https://www.vive.ucr.ac.cr/index.php/node/index/422 


\section{Información de las personas autoras}

Carlos Sandoval García. Docente e Investigador de la Universidad de Costa Rica, en el Doctorado en Ciencias Sociales sobre América Central, San José, Costa Rica. Dirección electrónica: carlos.sandoval@ucr.ac.cr, Orcid https://orcid.org/0000-0002-7660-4375

José Rivera Monge. Universidad de Costa Rica, en la Oficina de Registro e Información, San José, Costa Rica. Dirección electrónica: jose.rivera@ucr.ac.cr, Orcid https://orcid.org/0000-0001$\underline{7397-4701}$

Tatiana Carmona Rizo. Universidad de Costa Rica, en la Vicerrectoría de Vida Estudiantil, San José, Costa Rica. Dirección electrónica: jessica.carmonarizo@ucr.ac.cr, Orcid https://orcid.org/0000-0002-8122-2291

Magaly Jiménez Fernández. Universidad de Costa Rica, en la Vicerrectoría de Vida Estudiantil, San José, Costa Rica. Dirección electrónica: magaly.jimenez@ucr.ac.cr, Orcid https://orcid.org/0000-0002-6948-8409

María Isabel Sánchez Jiménez. Universidad de Costa Rica, en la Oficina de Orientación, San José, Costa Rica. Dirección electrónica: maria.sanchezjimenez@ucr.ac.cr, Orcid https://orcid.org/0000-0001-5288-2058

Sedali Solís Agüero. Universidad de Costa Rica, en la Oficina de Bienestar y Salud, San José, Costa Rica. Dirección electrónica: sedali.solis@ucr.ac.cr, Orcid https://orcid.org/0000-0003-4430$\underline{966 X}$

Lupita Abarca Espeleta. Universidad de Costa Rica, en el Programa de Voluntariado, San José, Costa Rica. Dirección electrónica: lupita.abarca@ucr.ac.cr Orcid https://orcid.org/0000-0003$\underline{3193-6461}$

María del Carmen Rojas Alfaro. Universidad de Costa Rica, en el Programa de Liderazgo, San José, Costa Rica. Dirección electrónica: maria.rojasalfaro@ucr.ac.cr Orcid https://orcid.org/0000$\underline{0002-6459-873 X}$

Anabelle León Chinchilla. Universidad de Costa Rica, en la Oficina de Becas y Atención Socioeconómica, San José, Costa Rica. Dirección electrónica: anabelle.leon@ucr.ac.cr Orcid https://orcid.org/0000-0001-7719-2918 
Revista indizada en

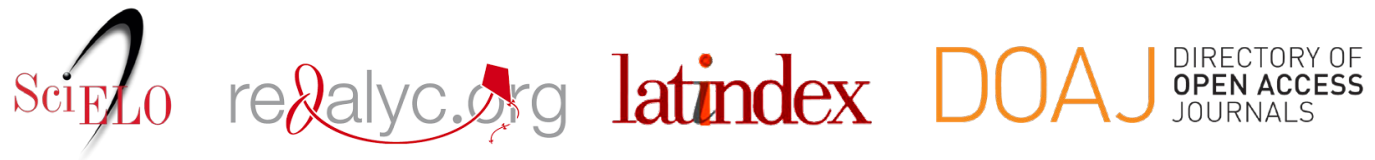

Distribuida en las bases de datos:

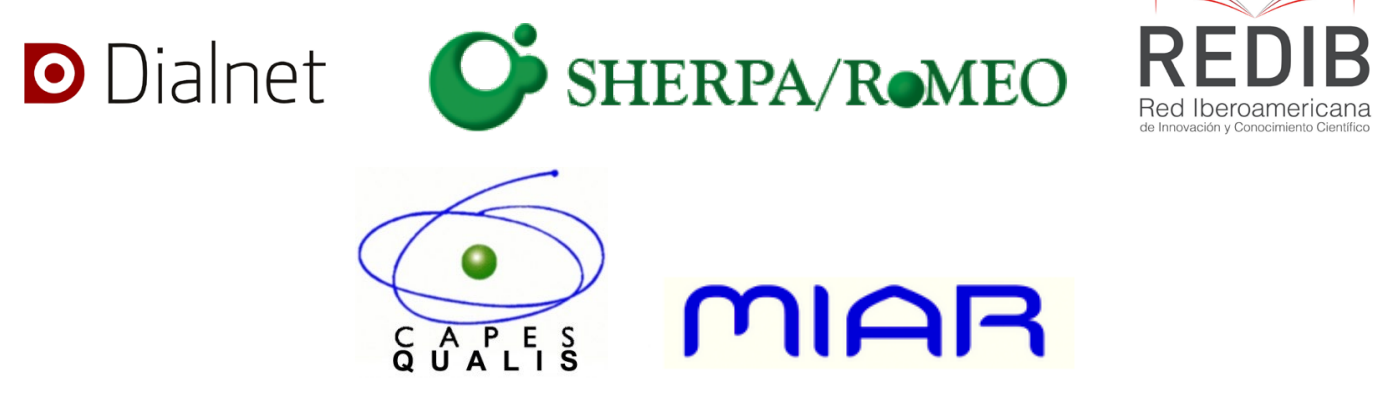

